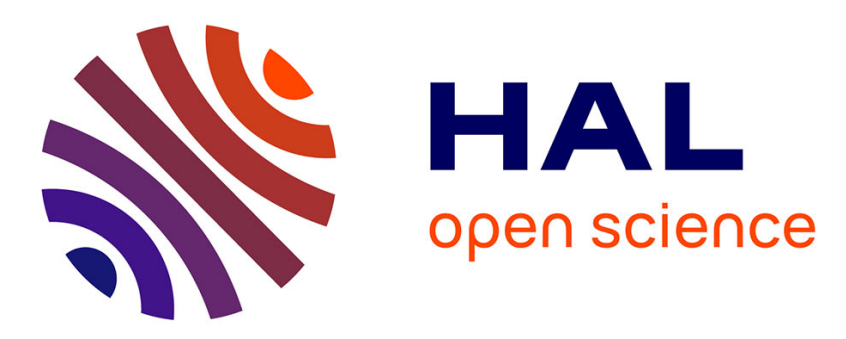

\title{
From volume medical images to quadratic surface patches
}

Ivan Bricault, Olivier Monga

\section{To cite this version:}

Ivan Bricault, Olivier Monga. From volume medical images to quadratic surface patches. [Research Report] RR-2380, INRIA. 1994. inria-00074297

\section{HAL Id: inria-00074297 https://hal.inria.fr/inria-00074297}

Submitted on 24 May 2006

HAL is a multi-disciplinary open access archive for the deposit and dissemination of scientific research documents, whether they are published or not. The documents may come from teaching and research institutions in France or abroad, or from public or private research centers.
L'archive ouverte pluridisciplinaire HAL, est destinée au dépôt et à la diffusion de documents scientifiques de niveau recherche, publiés ou non, émanant des établissements d'enseignement et de recherche français ou étrangers, des laboratoires publics ou privés. 



\title{
From volume medical images to quadratic surface patches
}

\author{
Ivan BRICAULT, Olivier MONGA \\ Programme 4 - Robotique, image et vision \\ Projet SYNTIM
}

Rapport de recherche $\mathrm{n}^{\circ} 2380$ - September 1994 - 34 pages

\begin{abstract}
In this paper, we show how to extract reliable informations about the shape of $3 \mathrm{D}$ objects, obtained from volume medical images. We present an optimal region-growing algorithm, that makes use of the differential characteristics of the object surface, and achieves a stable segmentation into a set of patches of quadratic surfaces. We show how this segmentation can be used to recognize and locate a target sub-structure on a global anatomic structure.
\end{abstract}

Key-words: Volume medical images, surface modeling, curvatures, crest lines, extremal mesh, optimal region growing, quadratic surfaces

(Résumé : tsvp) 


\section{Représentation paramétrique de surfaces à partir d'images médicales volumiques}

Résumé : Dans ce rapport, nous montrons comment extraire des informations fiables sur la forme d'objets $3 \mathrm{D}$, issus d'images médicales volumiques. Nous présentons un algorithme de croissance de régions optimal, qui utilise les caractéristiques différentielles de la surface de l'objet, et réalise une segmentation stable en morceaux de quadriques. Nous montrons comment cette segmentation peut servir à reconnaitre et localiser un élément anatomique particulier sur une structure de plus grande taille.

Mots-clé : Images volumiques 3D médicales, modélisation de surfaces, courbures, lignes de crêtes, extrêmalité gaussienne, surface quadrique, croissance de régions optimale 


\section{Introduction}

When processing volume medical images, a major task is to extract particular 3D structures automatically. These structures are represented by homogeneous areas [?, ?]. 3D edge detection provides points forming the boundaries of these structures, i.e. a discrete trace of the anatomical surfaces [?, ?, ?]. Some recent algorithms achieve a robust computation of the curvatures and of typical features of these surfaces such as crest lines, parabolic lines, or extremal mesh [?, ?, ?, ?]. In many cases these typical differential features provide only a partial description of the surface shape that is not sufficient for many applications, such as extraction of shape parameters, localization, registration... This paper deals with the next surface modeling stage: approximating the surfaces using parametric surface models. We choose to use quadric surface patches. We will discuss on the pertinence of this choice in section 2. Our work originates from the method described in [?, ?] to segment range data into planar and quadratic patches. The basic idea of $[?, ?]$ is to use a region growing scheme controlled by errors of quadric approximations.

The key contribution of our approach is to use also pre-computed differential features and particularly crest lines [?, ?], extremal mesh [?] and parabolic lines to guide the surface growing. Thus, we avoid partially the approximation instability arising when modeling complex objets, with many curvature discontinuities. We also use an optimal region growing algorithm described in [?] instead of the sub-optimal strategy presented in [?].

We come up with a surface growing algorithm controlled by both the approximation error and typical differential features. The final result is a description of the surface in terms of quadric patches. Experimental results on real medical data show clearly that this representation is very stable even for complex organic shapes. This stability is mainly due to the use of differential features to control the surface fitting. These quadric patches may be used for registration, localization, or recognition. We present some application examples.

\section{Motivation}

Let $I(x, y, z)$ be the grey level function representing a volume image (for instance see figure 1 ).

RR $n^{\circ} 2380$ 


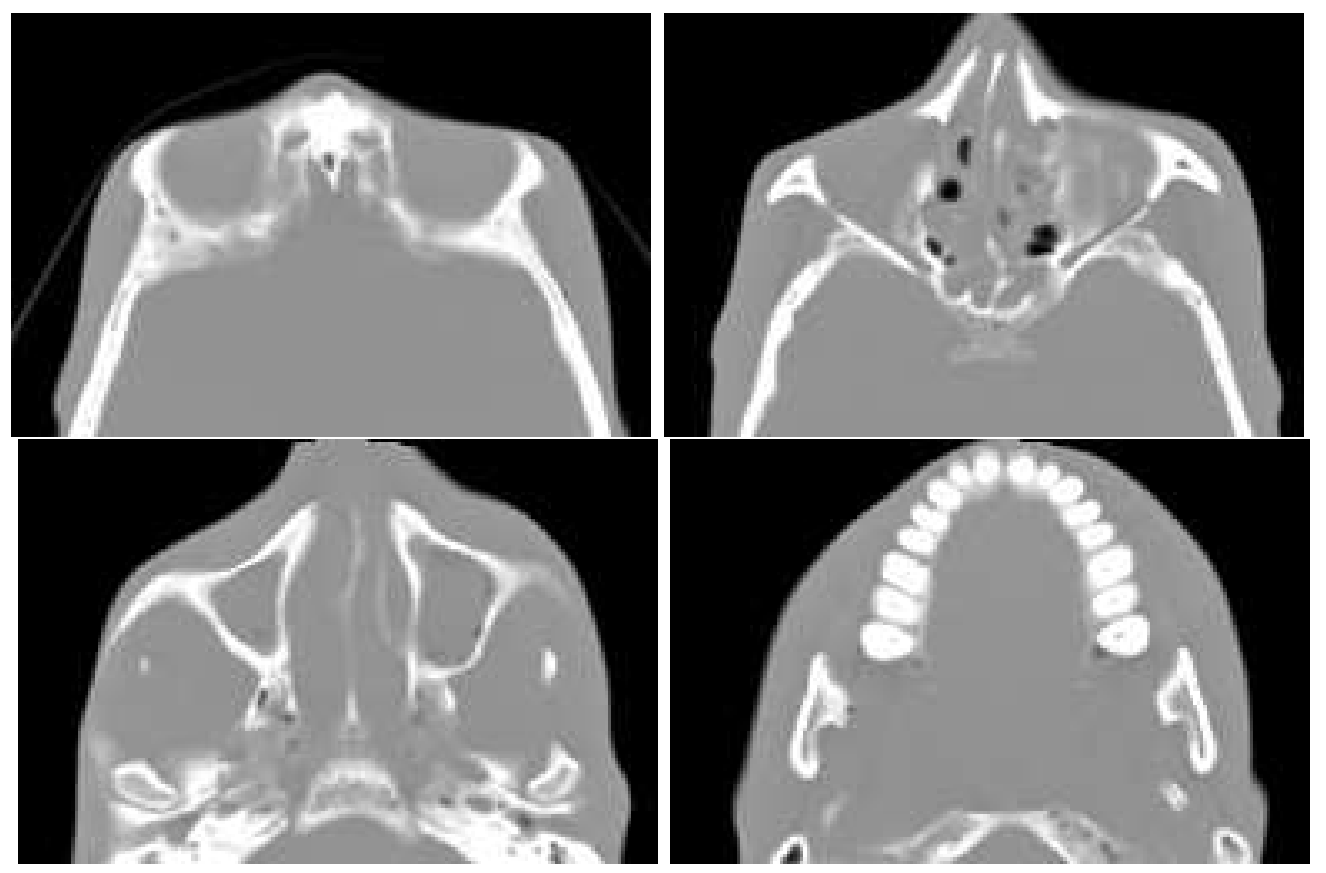

Figure 1: Cross sections of a X-scanner image of the skull (size of the image : $192 \times 128 \times 151$ )

Let $\operatorname{Edge}(x, y, z)$ be the result of a 3D edge detection algorithm (for instance see figure 2). $\operatorname{Edge}(x, y, z)$ is set to 1 for each edge point and to 0 otherwise.

Edge $(x, y, z)$ defines a set of points $E=\left\{M_{1}, M_{2} \ldots . M_{n}\right\}$ forming the discrete trace of a surface (for instance the skull surface in figure 2).

Using the algorithm described for instance in reference [?] we can extract typical features such as crest lines (see figure 3 ). These characteristic lines provide a partial but intrinsic representation of the surface which may be used for registration [?] or recognition.

Of course, these differential features are only a partial description of the surface. If we are interested about surface parts where there is no crest lines or about shape parameters, we have to deal also with some parametric surface representation. The problem here and particularly for medical data is that natural shapes do not follow a given parametric model. However, it is reasonable to approximate many natural surface shapes using primitives such as planes, quadrics [?], hyperquadrics [?, ?]. If we are concerned with a non planar piece-wise world, which is the case in medical data, quadrics or hyperquadrics are of course the most suitable. In the case of complex shapes, the whole surface cannot be approximated using a single hyperquadric. Moreover, the determination of a piece-wise approximation using 


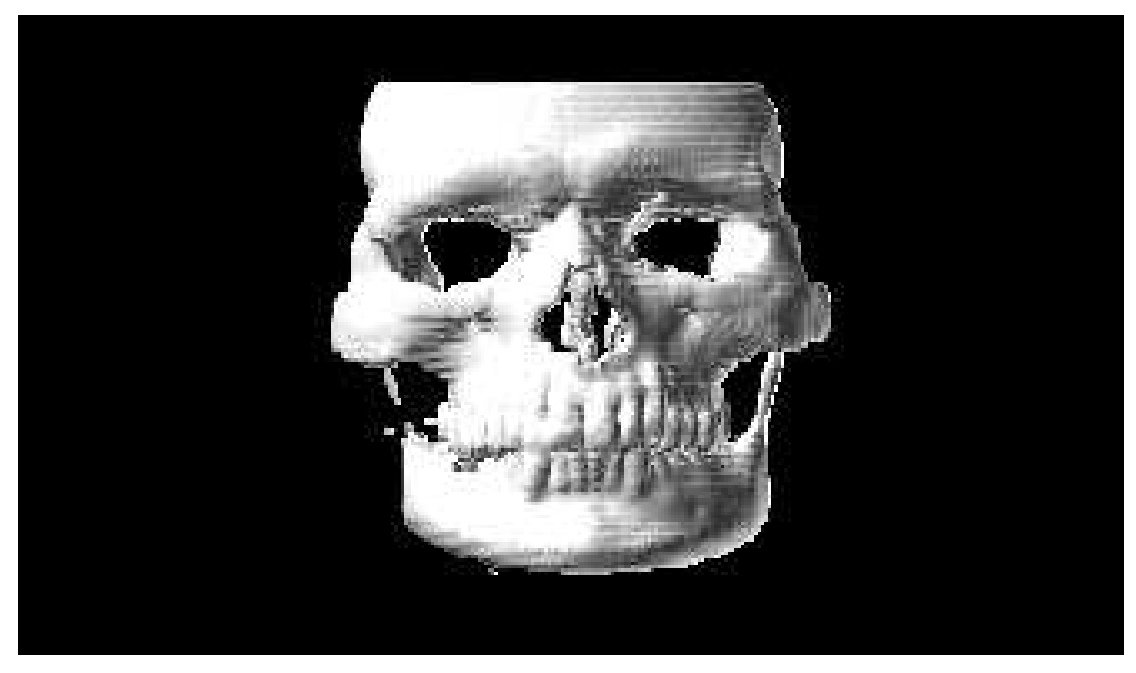

Figure 2: Data of figure 1 - Perpective view of the 3D edge map, provided by the algorithm described in reference [?]
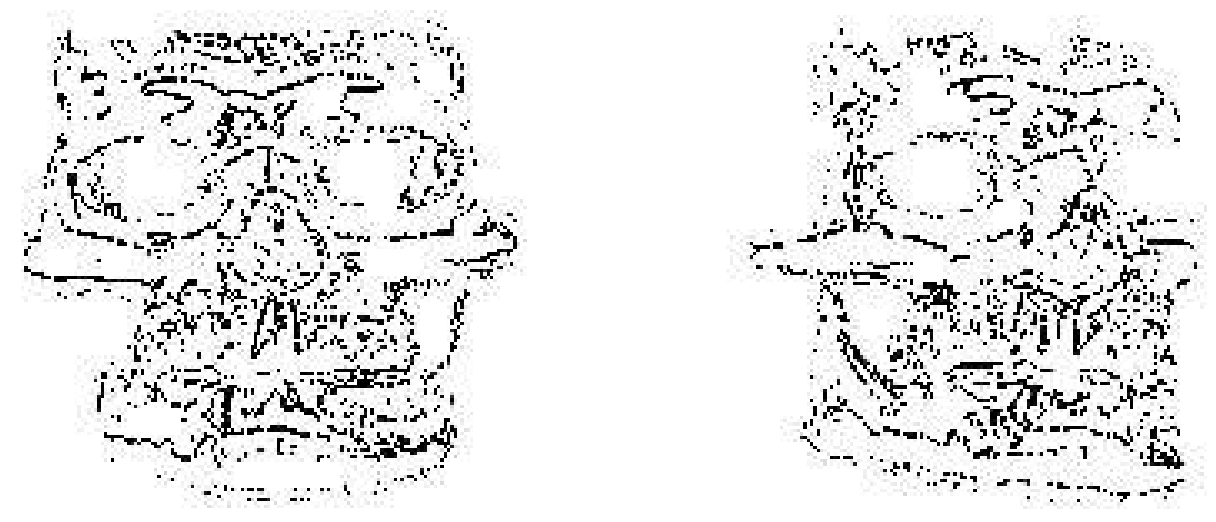

Figure 3: Skull of figure 2 - Perspective views of the crest lines obtained on two images taken at two different positions 
hyperquadric patches sets hard computational and robustness problems, arising from the model complexity. Thus, we choose a piece-wise approximation using quadratic patches such as in [?].

\section{Problem statement}

Following the notations of the previous section, we are looking for a partition of $E$ into connex parts such that each part is well approximated by a quadric surface. Using the formalism proposed in [?, ?] this segmentation problem can be defined using an homogeneity predicate $P$ and a quality function $C$ in the following way :

We look for a segmentation $S=\left\{R_{1}, R_{2} \ldots R_{m}\right\}$ of $E$ such that :

1. $E=\cup R_{i}, \quad i \in[1 \ldots m]$

2. $R_{i}$ is connex, $\forall i \in[1 \ldots m]$

3. $P\left(R_{i}\right)=$ true, $\forall i \in[1 \ldots m]$

4. $P\left(R_{i} \cup R_{j}\right)=$ false, $\forall i \neq j$, for each pair $\left(R_{i}, R_{j}\right)$ of connected patches.

5. among all partitions verifying the previous conditions, $S$ minimizes a quality function $C$ defined on the set of partitions of $E$.

In order to define $P$ and $C$, we first recall some definitions about the approximation of a set of points using a quadric. More details can be found in [?, ?].

Let $(A, \vec{v})$ be a quadric defined as the set of points $P$ verifying equation $P^{t} A P+\left.\right|_{1} ^{P} \cdot v=0$ ( $A$ is the quadratic matrix of size $3 \times 3, \vec{v}$ is a vector of dimension 4 ). Let $R_{j}$ be a set of points $\left\{P_{i}=\left(x_{i}, y_{i}, z_{i}\right)\right\}$ that we want to approximate in the $3 \mathrm{D}$ space. We define the matrices :

- $\mathcal{B}=\sum_{i}\left(x_{i}^{2}, y_{i}^{2}, z_{i}^{2}, \sqrt{2} x_{i} y_{i}, \sqrt{2} y_{i} z_{i}, \sqrt{2} z_{i} x_{i}\right)^{t}\left(x_{i}^{2}, y_{i}^{2}, z_{i}^{2}, \sqrt{2} x_{i} y_{i}, \sqrt{2} y_{i} z_{i}, \sqrt{2} z_{i} x_{i}\right)$

- $\mathcal{C}=\sum_{i}\left(x_{i}^{2}, y_{i}^{2}, z_{i}^{2}, \sqrt{2} x_{i} y_{i}, \sqrt{2} y_{i} z_{i}, \sqrt{2} z_{i} x_{i}\right)^{t}\left(x_{i}, y_{i}, z_{i}, 1\right)$

- $\mathcal{D}=\sum_{i}\left(x_{i}, y_{i}, z_{i}, 1\right)^{t}\left(x_{i}, y_{i}, z_{i}, 1\right)$ 
We notice that the updating of these matrices can be done iteratively when a new point is added. Let then $T=\left(T_{1}, T_{2}, T_{3}, T_{4}, T_{5}, T_{6}\right)^{t}$ be the eigen-vector attached to the smallest eigen-value of $6 \times 6$ Matrix $: \mathcal{B}-\mathcal{C D}^{-1} \mathcal{C}^{t}$. The quadric $(A, \vec{v})$ which minimizes the least mean square criterion defined by :

$$
\sum_{i}\left(P_{i}^{t} A P_{i}+\left.\right|_{1} ^{P_{i}} \cdot v\right)^{2}
$$

is given by :

$$
\begin{gathered}
A=\left[\begin{array}{ccc}
T_{1} & T_{4} / \sqrt{2} & T_{5} / \sqrt{2} \\
T_{4} / \sqrt{2} & T_{2} & T_{6} / \sqrt{2} \\
T_{5} / \sqrt{2} & T_{6} / \sqrt{2} & T_{3}
\end{array}\right] \\
\text { and } v=-\mathcal{D}^{-1} \mathcal{C}^{t} T .
\end{gathered}
$$

We notice that this criterion is not euclidean such as the optimal one proposed in [?]. But surface segmentation requires a criterion having strong computational properties. In the case of surface growing, the updating facility is crucial and this criterion can be updated almost recursively.

Let $F_{q}\left(R_{i}\right)$ be the smallest eigen-value of matrix $\mathcal{B}-\mathcal{C D}^{-1} \mathcal{C}^{t} . F_{q}\left(R_{i}\right)$ defines the least mean square error when fitting $R_{i}$ with a quadric. We set :

$$
P\left(R_{i}\right)=\text { true } \Longleftrightarrow F_{q}\left(R_{i}\right) / n_{R_{i}}<s
$$

where $s$ is a threshold defining the maximum allowed fitting error, and $n_{R_{i}}$ is the number of points of $R_{i}$; and

$$
C(S)=C\left(R_{1}, R_{2}, \ldots R_{m}\right)=\sum_{i} F_{q}\left(R_{i}\right)
$$

The determination of the [one] solution minimizing the criterion $C$ defined above is an NP complete problem. Therefore, for common data size, only sub-optimal algorithms can have a reasonable complexity. Classicaly, we can use region (surface) growing or region splitting. We choose a region growing strategy because this approach is more suitable when no a priori information is available on the data shape.

We use the optimal graph based region growing algorithm described in the reference [?]. Then we need to define:

$\operatorname{RR} n^{\circ} 2380$ 
1. an initial surface segmentation : $S_{\text {init }}(E)=\left\{I_{1}, I_{2} \ldots I_{p}\right\}$;

2. a sequence of pairs : $\left(P_{1}, Q_{1}\right),\left(P_{2}, Q_{2}\right), \ldots\left(P_{l}, Q_{l}\right)$; where $P_{i}$ is a merging predicate defined on each pair of connected patches (regions), and $Q_{i}$ is a merging cost defined for a pair of patches;

3. a set of features attached to patches (node features) and to the boundary of two patches (arc features) such that the merging predicates and the cost functions can be easily expressed ;

4. features updating functions, used during merging.

Points 1 and 2 define the growing strategy which is classical [?]. Points 3 and 4 define conditions allowing to achieve a very fast implementation of the region growing scheme, which is the main interest of the reference [?]. In our case, features are represented through matrices $\mathcal{B}, \mathcal{C}, \mathcal{D}$. In the sequel we will only deal with the points 1 and 2 ; the adequation of our predicates and cost functions to the data structure of [?] can be easily checked.

The region (surface) growing algorithm is the following one :

$\mathrm{S}=S_{\text {init }}(E)$

for $i=1$ to $l$ do

While a pair of connected regions whose union verifies $P_{i}$ exists do

- merge, among all the pairs $\left(R_{p}, R_{q}\right)$ whose union verifies $P_{i}$, the one such that $Q_{i}\left(R_{p} \cup R_{q}\right)$ is optimal

- update $S$

endwhile

endfor

Therefore, we have reduced our segmentation problem to the search of an initial segmentation algorithm and of adequate pairs $\left(P_{1}, Q_{1}\right),\left(P_{2}, Q_{2}\right), \ldots .\left(P_{l}, Q_{l}\right)$. In most region growing algorithms $[?, ?, ?]$ 
only homogeneity merging criteria are used. Here, the most natural homogeneity and merging criteria are :

$$
\begin{gathered}
P\left(R_{i}, R_{j}\right)=\text { true } \Longleftrightarrow F_{q}\left(R_{i} \cup R_{j}\right) / n_{R_{i} \cup R_{j}}<s \\
Q\left(R_{i}, R_{j}\right)=F_{q}\left(R_{i} \cup R_{j}\right) / n_{R_{i} \cup R_{j}}
\end{gathered}
$$

These are the criteria used in [?]. They provide a partition verifying points $1,2,3,4$ of the proposed definition of segmentation, but can hardly meet the point 5 which is crucial for the stability of the result. The robustness problems come mainly from the instability of the surface fitting when taking into account points where the curvatures present singularities (break points). A typical case is when signs of gaussian curvatures change. In order to overcome this key problem, we propose to define merging predicates and costs that use typical differential singularities such as : crest lines, extremal mesh, parabolic lines... The initialization of the surface growing is also an important stage that we tackle using differential singularities. In the next two sections we describe our initialization algorithm which provides an initial partition of the surface in quadric patches, and some merging strategies using extremal mesh and parabolic lines.

\section{Typical surface features}

\subsection{Extremal mesh}

In the references [?, ?] we propose a definition of the crest lines using partial derivatives of order $1,2,3$ of the image $I(x, y, z)$. We come up to the following expression which is the derivative of the maximum curvature along the maximum curvature direction. Let $\vec{t}_{1}$ be the maximum curvature direction, the derivative of the curvature in the direction $\overrightarrow{t_{1}}$ is :

$$
\nabla_{\overrightarrow{t_{1}}} k_{t_{1}}(m)=\frac{\|\vec{g}\|^{2} t_{1}^{T} \cdot\left[\begin{array}{c}
t_{1}^{T} H_{x} t_{1} \\
t_{1}^{T} H_{y} t_{1} \\
t_{1}^{T} H_{z} t_{1}
\end{array}\right]-\left(t_{1}^{T} H t_{1}\right)\left(t_{1}^{T} H g\right)}{\|\vec{g}\|^{3}}
$$

$\mathrm{RR} \mathrm{n}^{\circ} 2380$ 
where $\vec{g}$ is the gradient, $\mathrm{H}$ the hessian of $\mathrm{I}$ and $H_{x}, H_{y}, H_{z}$ its partial derivatives.

$$
\begin{gathered}
\vec{g}=\left[\begin{array}{c}
I_{x} \\
I_{y} \\
I_{z}
\end{array}\right] \\
H=\left[\begin{array}{ccc}
I_{x x} & I_{x y} & I_{x z} \\
I_{y x} & I_{y y} & I_{y z} \\
I_{z x} & I_{z y} & I_{z z}
\end{array}\right] \\
H_{x} \frac{\partial H}{\partial x}=\left[\begin{array}{ccc}
I_{x x x} & I_{x y x} & I_{x z x} \\
I_{y x x} & I_{y y x} & I_{y z x} \\
I_{z x x} & I_{z y x} & I_{z z x}
\end{array}\right]
\end{gathered}
$$

$\nabla_{\overrightarrow{t_{1}}} k_{t_{1}}(m)$ is called the "extremality criterion" (EC) and its zero-crossings correspond to crest lines. Although the zero-crossings of EC are (euclidean) invariant, only the magnitude of EC is invariant due to the fact that orientation of $\overrightarrow{t_{1}}$ is not defined. This problem arises when computing the zero-crossings of EC from a sign image. Although the coherence of orientations of $\overrightarrow{t_{1}}$ can be almost achieved practically $[?, ?, ?]$, it remains some cases where false zero-crossings are extracted. This was one of the motivations why Thirion introduced recently the Gaussian extremality which is defined as :

$$
G=\nabla_{\overrightarrow{t_{1}}} k_{t_{1}}(m) \nabla_{\overrightarrow{t_{2}}} k_{t_{2}}(m)
$$

with $\overrightarrow{t_{1}}$ and $\overrightarrow{t_{2}}$ being respectively parallel to the maximum and to the minimum curvature directions. $\overrightarrow{t_{1}}$ and $\overrightarrow{t_{2}}$ are oriented such that $\left(\vec{n}, \overrightarrow{t_{1}}, \overrightarrow{t_{2}}\right)$ is a direct orthonormal frame. $G$ is an euclidean differential invariant whose zero-crossings correspond to the points where its sign changes. The partition of a surface, in sets of connected points where the sign of $G$ is the same, is called the extremal partition (EP) [?]; and the lines forming the boundaries constitute the extremal mesh (EM) [?]. Therefore EP defines a segmentation of a surface into "smooth" patches, which do not include any points where the curvature is locally extremum. 


\subsection{Parabolic lines}

The sign of the Gaussian curvature defines a partition of a surface into connected sets of elliptic points (the gaussian curvature is positive) and hyperbolic points (the gaussian curvature is negative) [?]. The lines forming the boundary of this partition are called parabolic lines. Such lines can be extracted from 3D images using the algorithms described in [?].

The sign of the gaussian curvature is constant for all the points of a quadric surface (positive for an ellipsoid, negative for a hyperboloid, zero for a paraboloid). Figure 4 illustrates this remark.

\section{$5 \quad$ Using typical surface features for region growing}

\subsection{Region growing initialization}

Whatever is the region growing heuristics, the final result depends on the initial partition. Here, the problem is to find an initialization algorithm which provides intrinsic patches well approximated by quadric surfaces, and also with enough points to be significant. We propose to use the partition defined by maximal connected patches such that signs of the gaussian extremality and curvature are constant. Using the segmentation definition proposed precedently, this is equivalent to use the homogeneity predicate $P$ such that :

$$
P\left(R_{i}\right)=\text { true } \Longleftrightarrow\left[\forall(m, p) \in{R_{i}}^{2} ; \operatorname{Sign}(g(m))=\operatorname{Sign}(g(p)) \wedge \operatorname{Sign}(G(m))=\operatorname{Sign}(G(p))\right]
$$

where $g(m), g(p), G(m)$ and $G(p)$ are respectively the gaussian curvature and extremality at points $m$ and $p$. When applying a simple algorithm we obtain some patches having the following shape :

$\mathrm{RR} \mathrm{n}^{\circ} 2380$ 
Figure 4: Quadrics - hyperboloids, paraboloid, ellipsoid, hyperbolic paraboloid. 


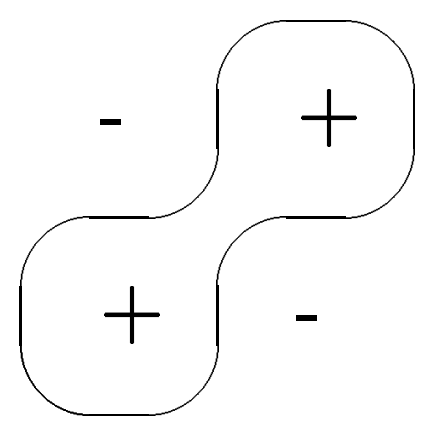

In this example, it is necessary to split the positive region in two parts. Otherwise, this bad initial partition would damage the region growing result. The use of simple tools coming from mathematical morphology solves this problem [?] :

- we choose a structuring element, typically a globe whose radius is 2 or 3 pixels ;

- we do an opening using this structuring element; this operation smooths and deconnects region parts where the diameter is below the one of the structuring element;

- using a geodesic dilation of each isolated components we grasp all points from the initial region.

This algorithm allows to segment the precedent figure into two positive regions instead of one.

We also use a mathematical morphology operator to define the boundaries between two regions : we select points located in the difference between a region and the geodesic dilation of the neighbouring region. Depending on the size of the structuring element, we build a border line or a border strip of a given width.

\subsection{Merging strategies}

\subsubsection{Fitting error}

By taking the notations of section 3 we define the fitting error $F_{q}(R)$ as the smallest eigenvalue of $\mathcal{B}-\mathcal{C D}^{-1} \mathcal{C}^{t}$. Then a straightforward merging strategy is to use the following merging 
predicate and merging cost [?] :

$$
\begin{gathered}
P\left(R_{1}, R_{2}\right) \Longleftrightarrow F_{q}\left(R_{1} \cup R_{2}\right) / n_{R_{1} \cup R_{2}}<s \\
Q\left(R_{1}, R_{2}\right)=F_{q}\left(R_{1} \cup R_{2}\right) / n_{R_{1} \cup R_{2}}
\end{gathered}
$$

where $s$ is a given threshold, and $n_{R_{1} \cup R_{2}}$ is the number of points in $R_{1} \cup R_{2}$.

The drawback of this merging strategy is that the continuity of the two patches is badly taken into account. This fact creates instabilities when merging two patches whose quadrics are discontinuous at the border. For instance, if we implement this strategy using a distorted cube and a planar model we obtain the six faces. But the boundaries of the planar patches are not correctly defined [See figure 5: one 2D-section of the cube $3 \mathrm{D}$-image is shown; highlighted points have been merged within a particular patch, and the line represents the approximating plane trace in this section].

Figure 5: Cube - Approximation of one side by a plane.

\subsubsection{Continuity fitting error of two patches}

A way to avoid instability around the discontinuities is to define a homogeneity criterion taking into account the similarity of the two quadrics. We propose the following criterion, which estimates how well one patch extends the other:

$$
\begin{gathered}
P\left(R_{1}, R_{2}\right) \Longleftrightarrow E_{R_{1}}\left(R_{2}\right) / n_{R_{2}}<s \wedge E_{R_{2}}\left(R_{1}\right) / n_{R_{1}}<s \\
Q\left(R_{1}, R_{2}\right)=\left[E_{R_{1}}\left(R_{2}\right)+E_{R_{2}}\left(R_{1}\right)\right] / n_{R_{1} \cup R_{2}}
\end{gathered}
$$


where $s$ is a given threshold and :

$$
E_{R_{1}}\left(R_{2}\right)=T_{2}^{t} \cdot\left(\mathcal{B}_{1}-\mathcal{C}_{1} \mathcal{D}_{1}^{-1} \mathcal{C}_{1}^{t}\right) \cdot T_{2}
$$

$E_{R_{1}}\left(R_{2}\right)$ is the fitting error when approximating patch $R_{2}$ by the quadric associated to $R_{1}$. $\mathcal{B}_{1}, \mathcal{C}_{1}, \mathcal{D}_{1}$ are defined for patch $R_{1}$ using the notations of section 3 and $T_{2}$ is the eigen-vector corresponding to the quadric attached to patch $R_{2}$.

$T_{2}^{t} \cdot\left(B_{1}-C_{1} D_{1}^{-1} C_{1}^{t}\right) \cdot T_{2}$ is the value at $T_{2}$ of the quadratic form whose value at $T_{1}$ is the approximation error of $R_{1}$. Numerically, this choice for the criterion calculation ensures the coherence of the error estimation.

Figure 6 illustrates the advantage of this "continuity criterion" versus the previous one.

Figure 6: Cube - Approximation of one side by a plane, using patch continuity criterion.

\subsubsection{Taking into account extremal points}

The criterion described precedently allows to obtain some good patches [see figure 7 first column ; from top to bottom, successive $2 \mathrm{D}$-sections of the $3 \mathrm{D}$-image are represented, and a particular patch is shown in black; the corresponding quadric surface lies in the transition between gray and white regions]. But these results are very sensitive to the threshold value and moreover coins and edges could be better approximated : see for instance how a threshold too high leads to one big patch for the whole cube (figure 7 second column).

A way to improve stability and localization is to introduce the fitting error for the extremal and parabolic points located on the boundary between two regions. The introduction of a 

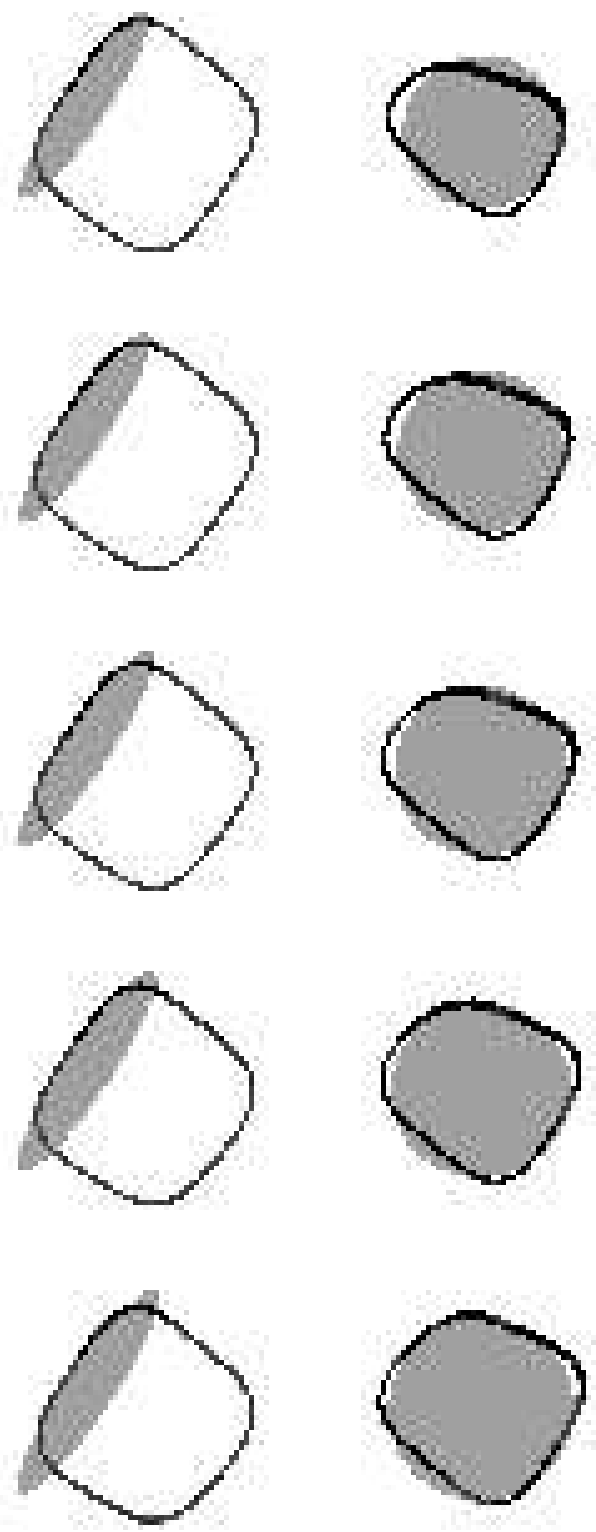

Figure 7: Approximation of the distorted cube by quadrics using patch continuity 
threshold on this error can guarantee that the merging maintains a good fitting for extremal and parabolic points.

Let us note $B_{o}\left(R_{1}, R_{2}\right)$ the region formed by the set of points of the border between $R_{1}$ and $R_{2}$. We notice that, due to our initialization algorithm, this boundary includes only differential singularities : extremal and parabolic points.

Let us note the fitting error at the boundary :

$$
E_{R_{1} \cup R_{2}}\left(B_{o}\left(R_{1}, R_{2}\right)\right)
$$

which is the approximation error of the boundary using the quadric attached to $R_{1} \cup R_{2}$.

Then we come up with the following merging strategy :

$$
\begin{aligned}
P\left(R_{1}, R_{2}\right) \Longleftrightarrow & E_{R_{1}}\left(R_{2}\right) / n_{R_{2}}<s_{c} \wedge E_{R_{2}}\left(R_{1}\right) / n_{R_{1}}<s_{c} \\
& \wedge E_{R_{1} \cup R_{2}}\left(B_{o}\left(R_{1}, R_{2}\right)\right) / n_{B_{o}\left(R_{1}, R_{2}\right)}<s_{b}
\end{aligned}
$$

where $s_{c}$ and $s_{b}$ are respectively the "continuity" threshold and the "boundary" threshold.

$$
Q\left(R_{1}, R_{2}\right)=E_{R_{1} \cup R_{2}}\left(B_{o}\left(R_{1}, R_{2}\right)\right) / n_{B_{o}\left(R_{1}, R_{2}\right)}+\left[E_{R_{2}}\left(R_{1}\right)+E_{R_{1}}\left(R_{2}\right)\right] / n_{R_{1} \cup R_{2}}
$$

\subsubsection{Relative error criterion}

The problem induced by the previous strategy is the determination of absolute thresholds $s_{c}$ and $s_{b}$, which are dependant of each particular application (and in particular when the noise on the data is not homogeneous). This difficulty can be overcome using relative thresholds (percentage of tolerance): we authorize the merging only if the increase of the error after merging is less than a given ratio of a local reference error.

The reference error $E_{r e f}\left(R_{i}\right)$ for patch $R_{i}$ is initialized by the fitting error $F_{q}\left(R_{i}\right)$ within initial region growing partition. Then $E_{r e f}$ is updated after a merging in the following way :

$$
E_{r e f}\left(R_{1} \cup R_{2}\right)=\text { Minimum of } E_{r e f}\left(R_{1}\right)+E_{r e f}\left(R_{2}\right) \text { and } F_{q}\left(R_{1} \cup R_{2}\right)
$$

We use an expression with a minimum to ensure that the reference error for a patch is the least error met so far.

$\mathrm{RR} \mathrm{n}^{\circ} 2380$ 


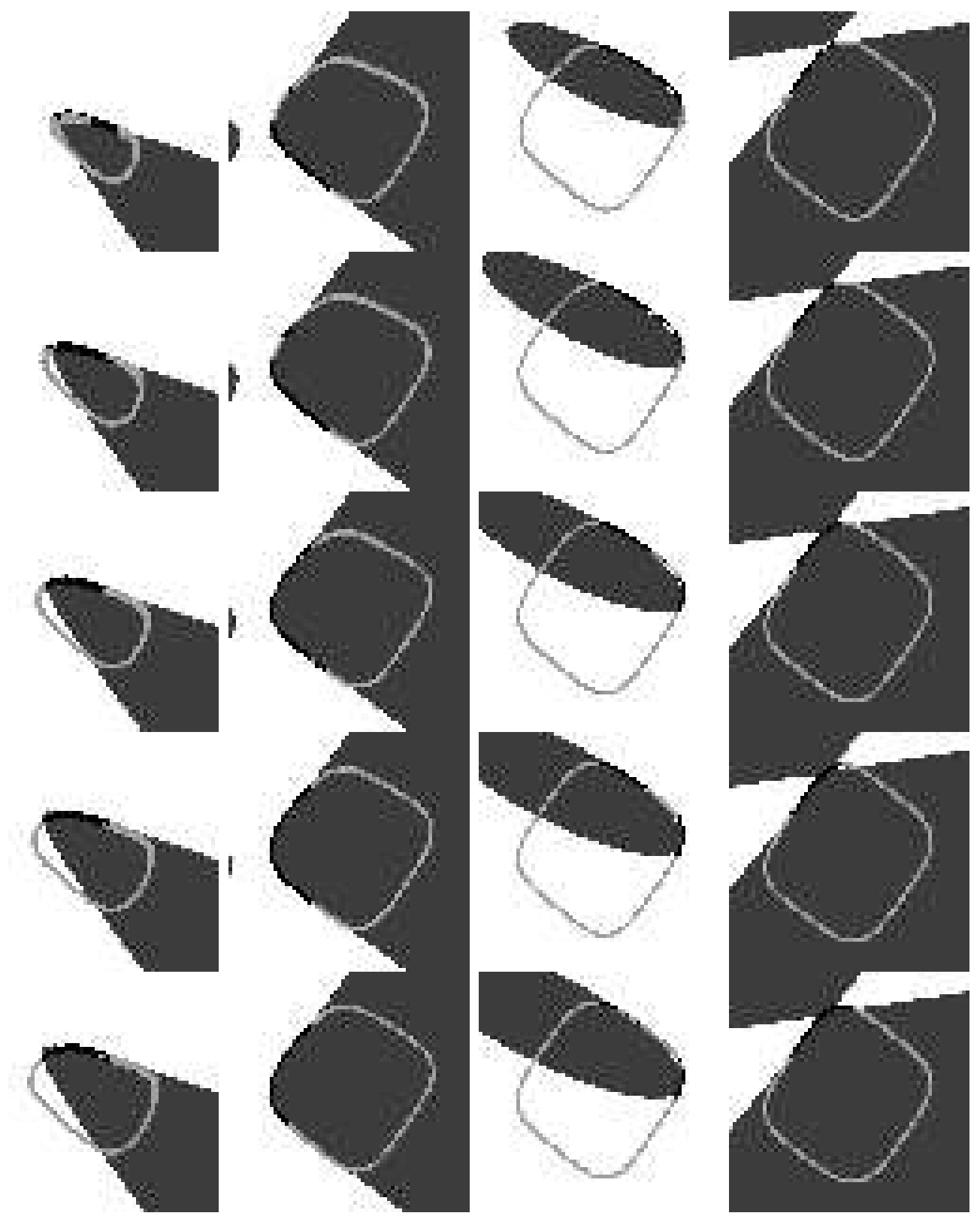

Figure 8: Approximation of the distorded cube using quadrics using a threshold of the fitting error at boundary points. 
Using an analogous scheme, we define the reference error for the border $E_{r e f}\left(B_{o}\left(R_{1}, R_{2}\right)\right)$. Here, the initialization value is :

$$
\left[E_{R_{1}}\left(B_{o}\left(R_{1}, R_{2}\right)\right)+E_{R_{2}}\left(B_{o}\left(R_{1}, R_{2}\right)\right)\right] / 2
$$

Figure 9 presents some results obtained using this heuristic.

\subsubsection{Merging heuristic}

Finally we come up with the grouping strategy defined by $P$ and $Q$ :

$$
\begin{gathered}
P\left(R_{1}, R_{2}\right)=\text { true } \Longleftrightarrow \quad\left[\left(E_{R_{1}}\left(R_{2}\right)<s_{c} \cdot E_{\text {ref }}\left(R_{2}\right)\right) \vee\left(\left(E_{R_{2}}\left(R_{1}\right)<s_{c} \cdot E_{\text {ref }}\left(R_{1}\right)\right)\right]\right. \\
\wedge\left(E_{R_{1} \cup R_{2}}\left(B_{o}\left(R_{1}, R_{2}\right)\right)<s_{b} \cdot E_{\text {ref }}\left(B_{o}\left(R_{1}, R_{2}\right)\right)\right) \\
Q_{1}\left(R_{1}, R_{2}\right)= \\
\\
\left.+E_{R_{1} \cup R_{2}}\left(B_{o}\left(R_{1}, R_{2}\right)\right) / n_{B_{o}\left(R_{1}, R_{2}\right)}\left(R_{2}\right)+E_{R_{2}}\left(R_{1}\right)\right] / n_{R_{1} \cup R_{2}}
\end{gathered}
$$

$s_{c}$ and $s_{b}$ are the continuity and boundary thresholds, defining the maximum authorized ratio for : merging error divided by reference error.

In order to improve this merging strategy, we also use more restrictive values for $s_{c}$ and $s_{b}$ when merging two regions of different gaussian nature (an elliptic and an hyperbolic region).

\section{Algorithm}

We come up with the following algorithm whose input is a $3 \mathrm{D}$ volume image and whose final result is a labelling of the iso-surfaces into quadric patches.

1. Extraction of $3 \mathrm{D}$ edges in a volume image $I(x, y, z)$ using the algorithm described in $[?]$;

2. computation of curvatures, parabolic lines and crest lines on the $3 \mathrm{D}$ edge map using the methods described in [?, ?];

$\operatorname{RR} n^{\circ} 2380$ 

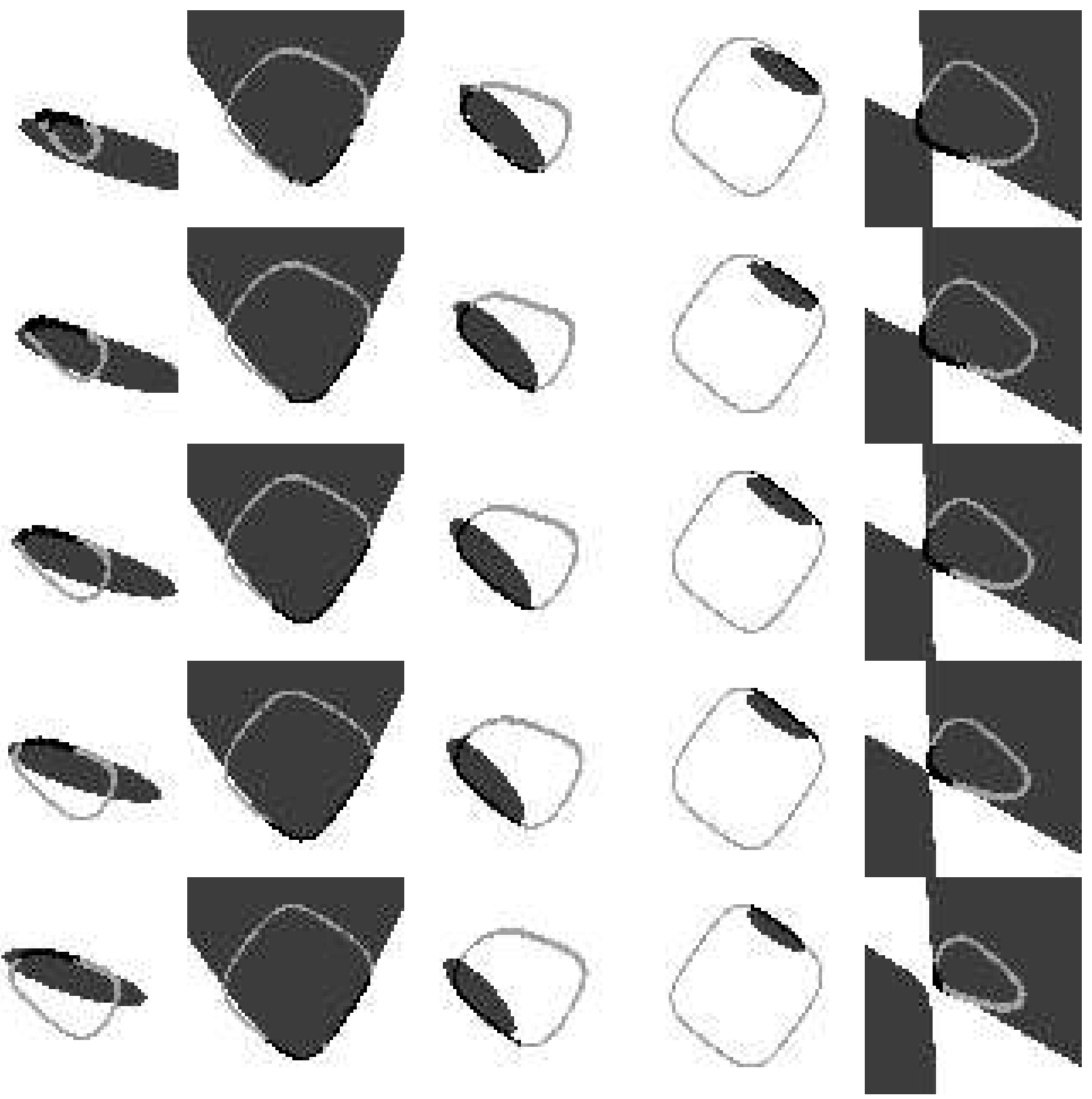

Figure 9: Approximation of the cube using quadrics - Method using relative error criteria. 
3. initial labelling of the 3D edge map into quadric patches using the extremal mesh [?] and the parabolic lines;

4. building of an adjacency valuated graph describing the initial labelling and use of the region (surface) growing strategy described in the previous section $(P, Q)$. This strategy is applied during various steps, corresponding to different values of the thresholds (less and less restrictive). The output is a labelling of the points of the $3 \mathrm{D}$ edge maps into connected patches, which are approximated using a quadric surface; this labelling is described by a valuated graph where each node is attached to a patch.

\section{Experimental results}

\subsection{Introduction}

As pointed out precedently, the final result of our sequence of algorithms is a partition of the 3D edge maps into sets of connected points (patches) that fit to a quadric surface. This partition is represented using a valuated adjacency graph where each node is attached to a patch and to the parameters of the corresponding quadric surface (these parameters are frame invariant [?]). This new data representation is interesting if it presents some stability properties. Of course the ideal property would be the invariance of all the quadric patches, that can hardly be achieved for natural shapes. So our goal is more to extract typical and useful informations about the shape than to describe it completely.

In order to test our approach we use two scanner $\mathrm{X}$ images of the same skull but taken at two different positions. Then we can check the stability of the results. We also illustrate the interest of this representation for the registration and for the recognition-locating of a target sub-structure on a global anatomic structure.

Figures 10 to 12 presents some results for images of a bottom jaw (light grey points). First columns reproduce successive slices from the first position 3D image; second columns correspond to second position. Points belonging to the particular patch shown are in black, and the corresponding quadric surface lies in the transition between white and dark grey regions.

RR $\mathrm{n}^{\circ} 2380$ 


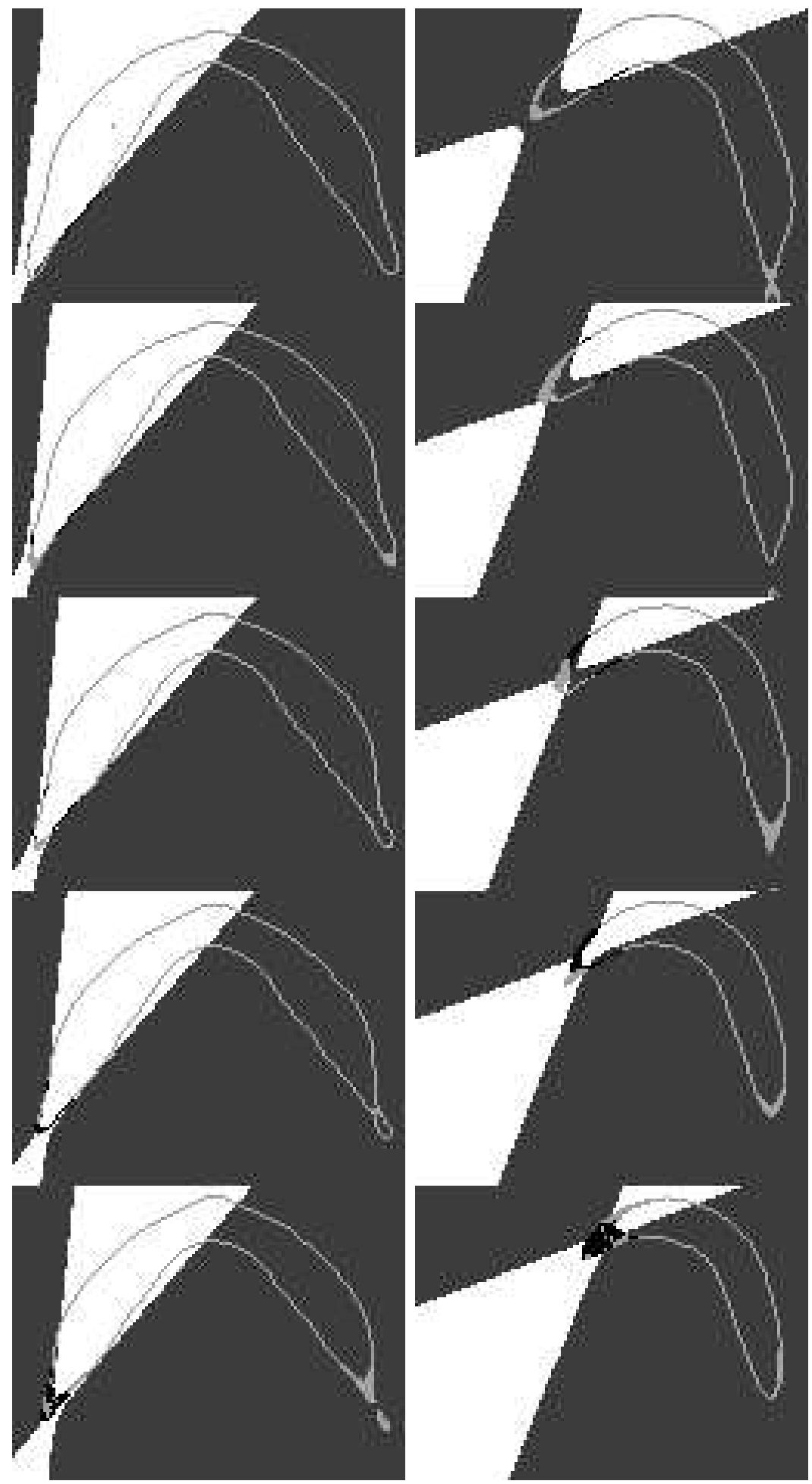

Figure 10: Approximation of a bottom jaw taken at two positions using quadrics - Patch on left posterior extremity. 

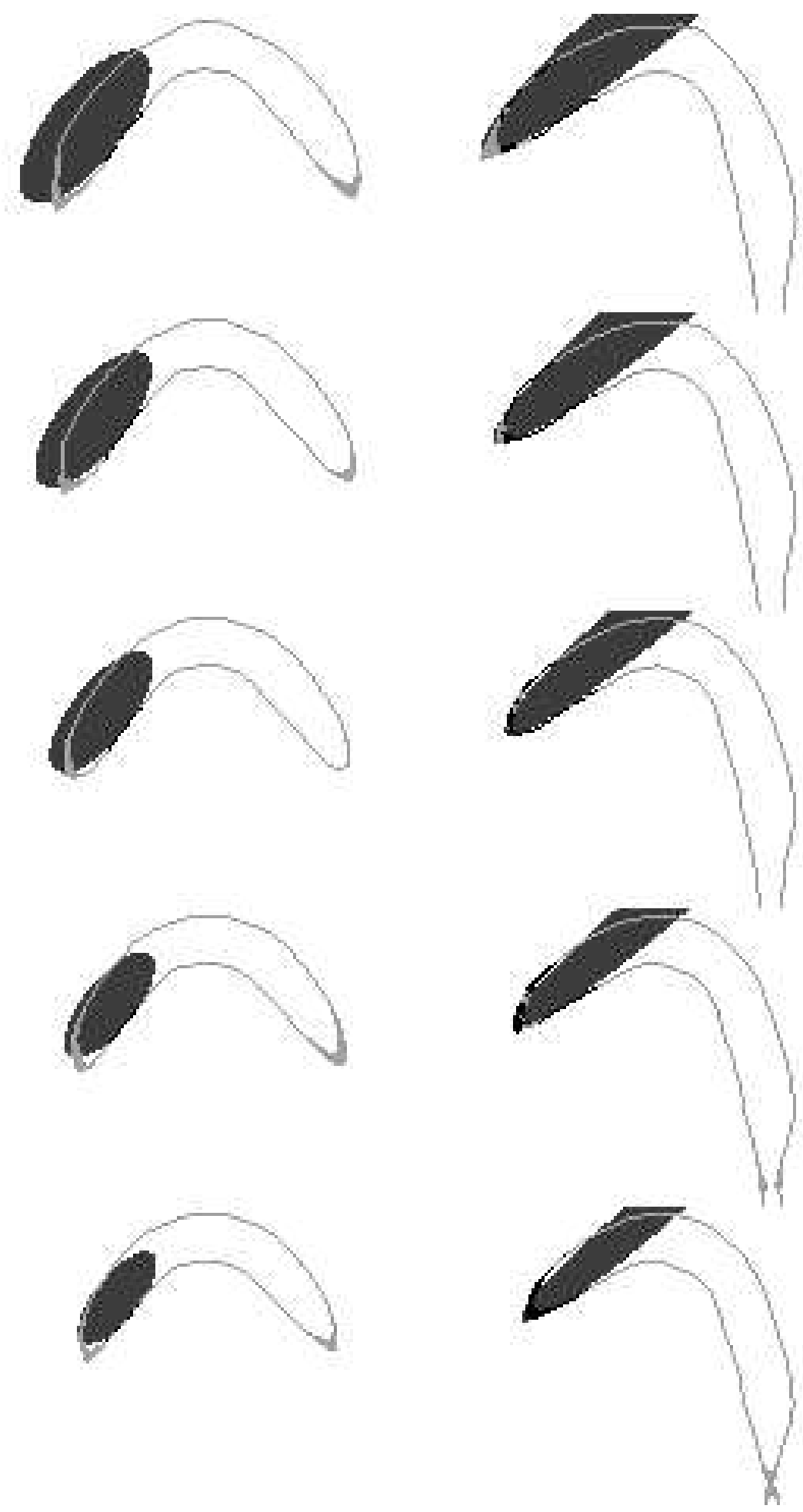

Figure 11: Approximation of a bottom jaw taken at two positions using quadrics - Patch on left posterior extremity. 


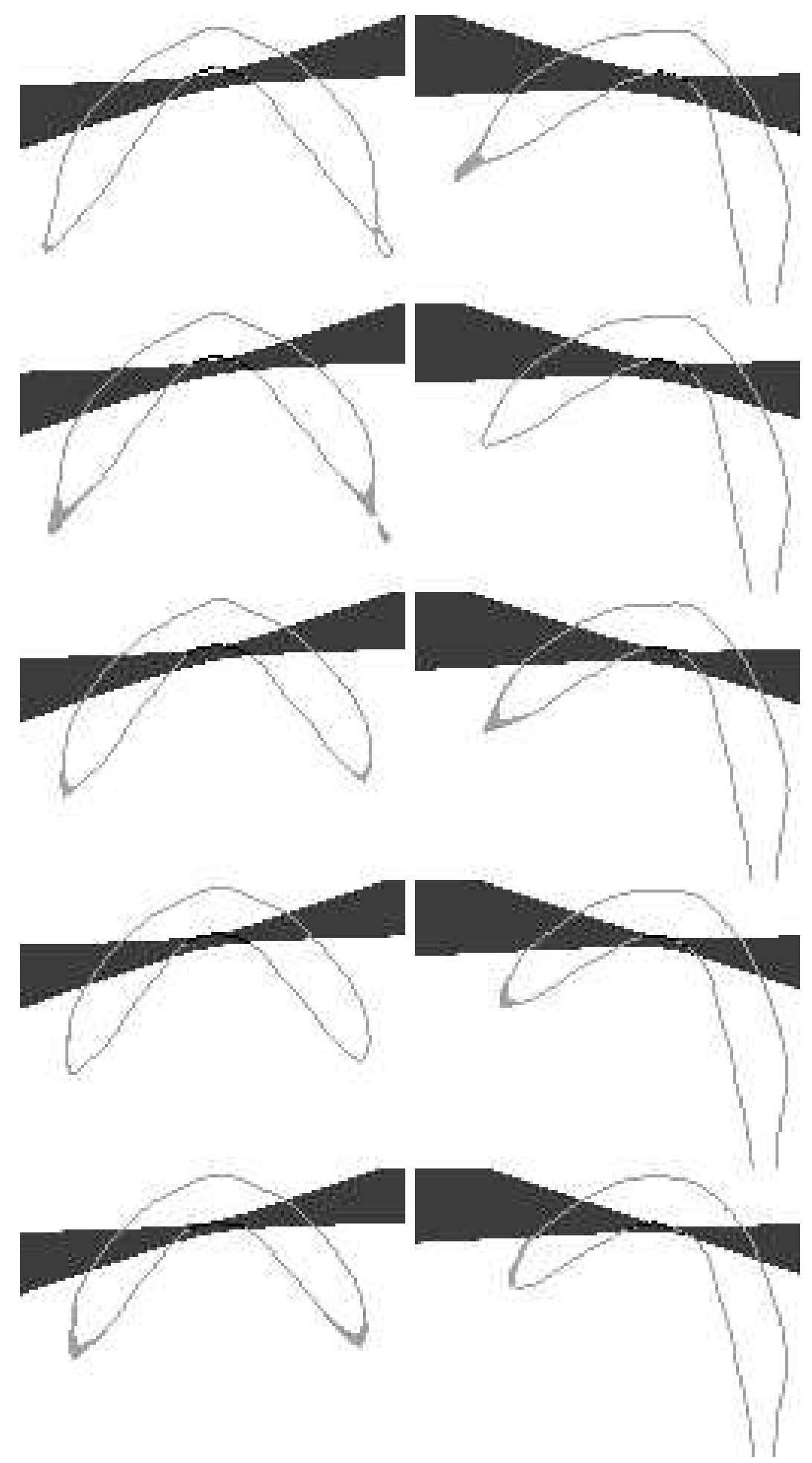

Figure 12: Approximation of a jaw taken at two positions using quadrics - Patch on front inner side. 


\subsection{Registration algorithm}

The segmentation into quadratic surfaces, presented above, helps us to achieve the matching process. We developped a registration algorithm according to the prediction-verification paradigm, with the following main features :

- From a set of matched patches, the displacement (represented by a quaternion) is estimated by superimposing the centroid of a patch and the centroid of the matched patch. This estimation uses a least mean square estimation of the best fitting quaternion $($ see $[?])$.

- Once a displacement $D$ has been estimated, we try to match a new pair of patches. We search for the pair of patches that are the nearest, according the following vicinity criterion : we compute the error $E_{D\left(R_{2}\right)}\left(R_{1}\right)$ when approximating the first patch by the quadratic surface of the second patch, after displacement; and we compare this error with the error $F_{q}\left(R_{1}\right)$ when approximating the first patch by its own quadratic surface. Thus, this criterion is much alike the continuity criterion introduced above.

This criterion makes use of the quadratic segmentation information, and is able to discriminate with a great accuracy among the possible matches, according to the position and shape of the different patches.

- For the initial estimation of a displacement, 3 matches are needed. They are guided by the similarity of parameters (eigenvalues and eigenvectors of the quadratic surfaces) for the possible patches.

Here we do not tackle the problem of a precise registration. Our aim is to rapidly recognize and locate (approximatively) a particular structure, despite of the following difficulties :

- we want to recognize and locate a particular substructure on a bigger structure; both can have thousands of edge points.

- we have no a-priori information about where the structure should be found

A more precise registration can follow the one we present; for example thanks to an iterative method using splines (see [?, ?]). This type of methods requires a good enough initial 
estimate, in order to avoid local minima; thus registration from quadratic segmentation can complete and extend application field for iterative methods.

\subsection{Registration results}

We consider here 2 volumic scanner images from the upper jaw of a patient, with 2 different orientations. The first orientation data include 15 successive sections; 5 sections are reproduced figure 13 (light grey points). Using our quadratic patches extraction algorithm, we obtained 5 patches of important size. Each of these patches is represented in one section on figure 13 : points of the structure belonging to the patch are in black, and the approximating quadratic surface is in dark grey. As a matter of fact, we performed a $3 \mathrm{D}$ approximation : each of the five quadratic surface follows the object surface along various successive sections, even though only one is represented.

In the same way, 12 sections define the upper jaw in the second orientation, and 5 of them are reproduced on figure 14 . We have also represented the 5 most important size extracted patches and the approximating quadratic surfaces.

Thanks to this extraction of quadratic surfaces, our algorithm is able to find all 4 pairs of corresponding patches between the two images. For instance, the third section on figure 13 is matched automatically with the fourth section figure 14, because both patches represent the inner side of teeth. Patches with no equivalent (figure 13 last section and figure 14 third section) are automatically identified and put away : this fact shows the pertinence of the matching criterion. This criterion is reliable in all cases, even though a few matched quadratic surfaces have different appearances - because they give a good representation for the shape of stable patches anyway.

Besides the algorithm is able to achieve the registration; the result is presented on figure 15 . 


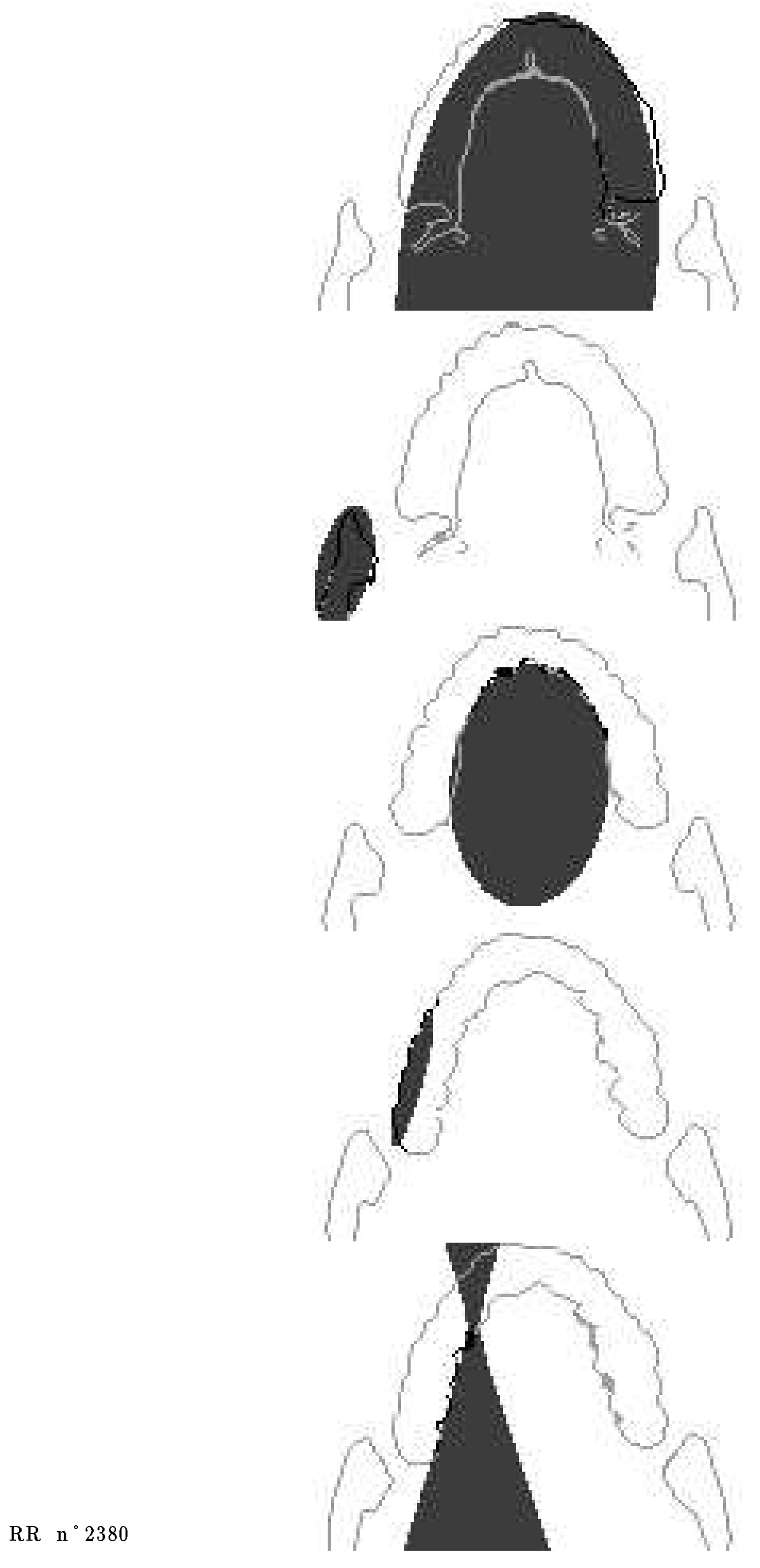

Figure 13: 5 sections from upper jaw, first orientation. 

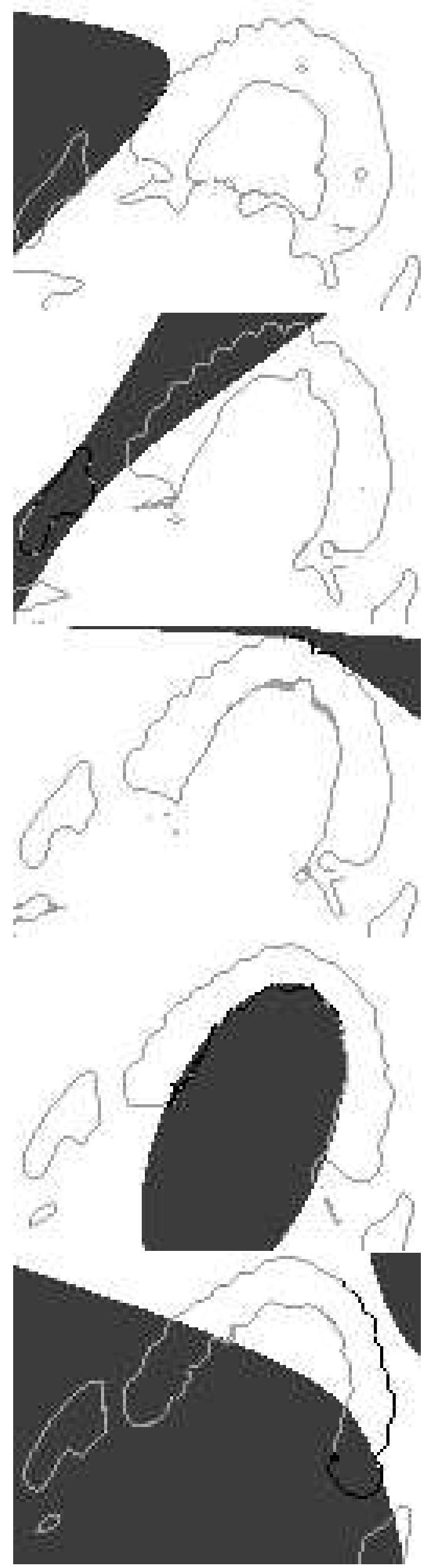

Figure 14: 5 sections from upper jaw, second orientation. 


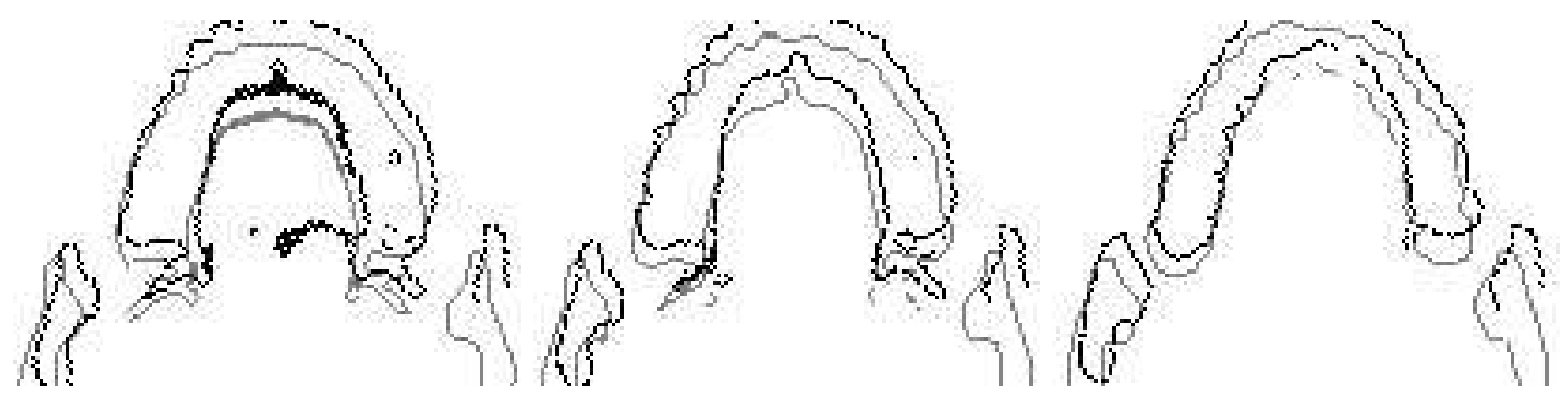

Figure 15: 3 sections from images of upper jaw, after registration of second orientation (black points) on first orientation (grey points).

\subsection{Recognition and locating results}

We focus now on the problem of recognizing and locating a target substructure on a global anatomic structure. The practical application field could be the computer-assisted guidance of a surgical act.

\subsubsection{Forehead}

Here, a volumic scanner image including 26 successive sections defines the target substructure (the forehead). Five sections are reproduced on figure 16. Using our quadratic patches extraction algorithm, we obtained 5 patches of important size. Each of these patches is represented in one section figure 16.

We can observe that the various patches are associated with a particular anatomic component in the forehead : inner and outer side of left part, inner and outer side of middle part, sinus.

Besides, a volumic scanner image including 47 successive sections defines the global structure (the top of skull). Seven sections are reproduced figure 16. Using our quadratic patches extraction algorithm, we obtained 9 patches of important size. Seven of these patches are represented in figure 17. We can recognize the same extracted anatomic components as before; and we add the eye-socket and the back side of eye.

$\mathrm{RR} \mathrm{n}^{\circ} 2380$ 


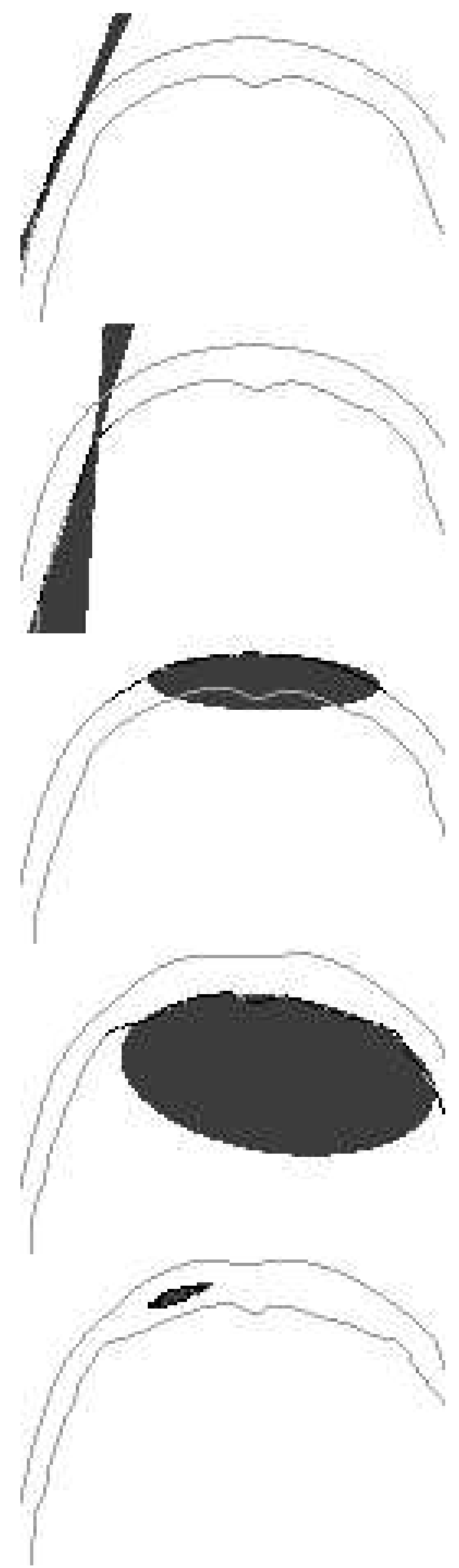

Inria

Figure 16: Forehead -5 sections from the target substructure. 

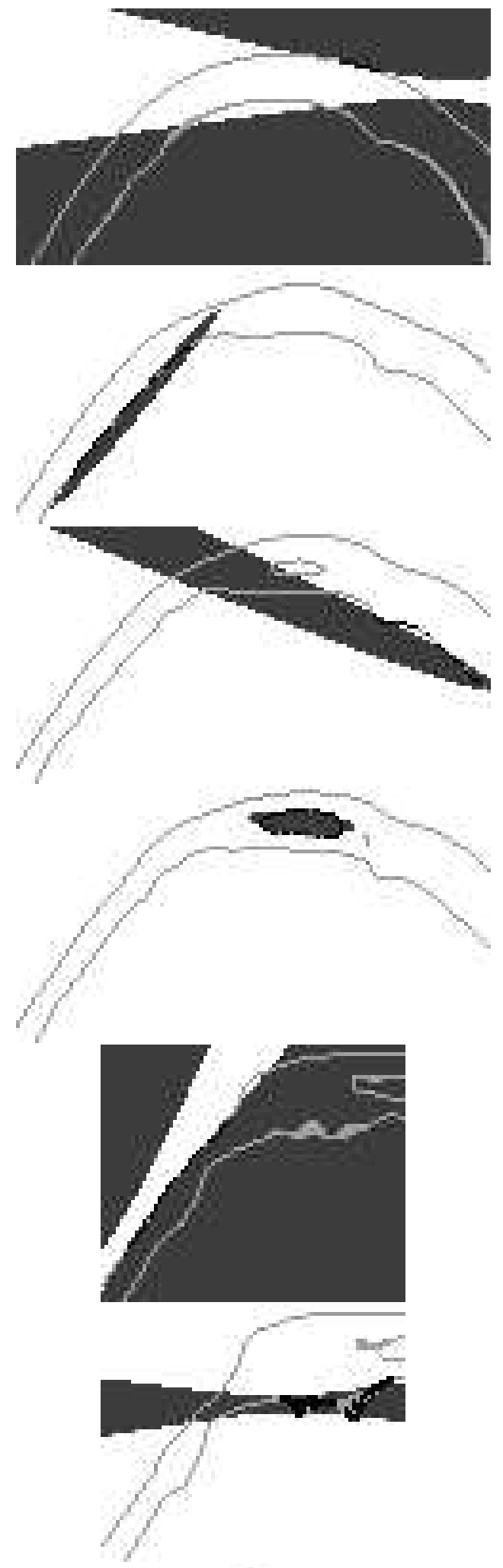

$\mathrm{RR} \mathrm{n}^{\circ} 2380$

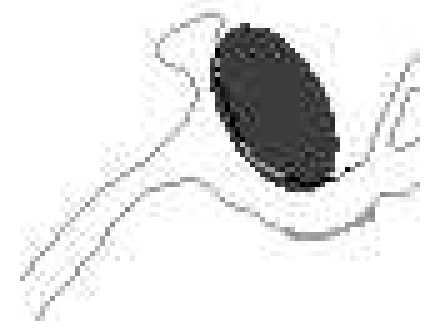

Figure 17: Global structure -7 sections from top skull. 

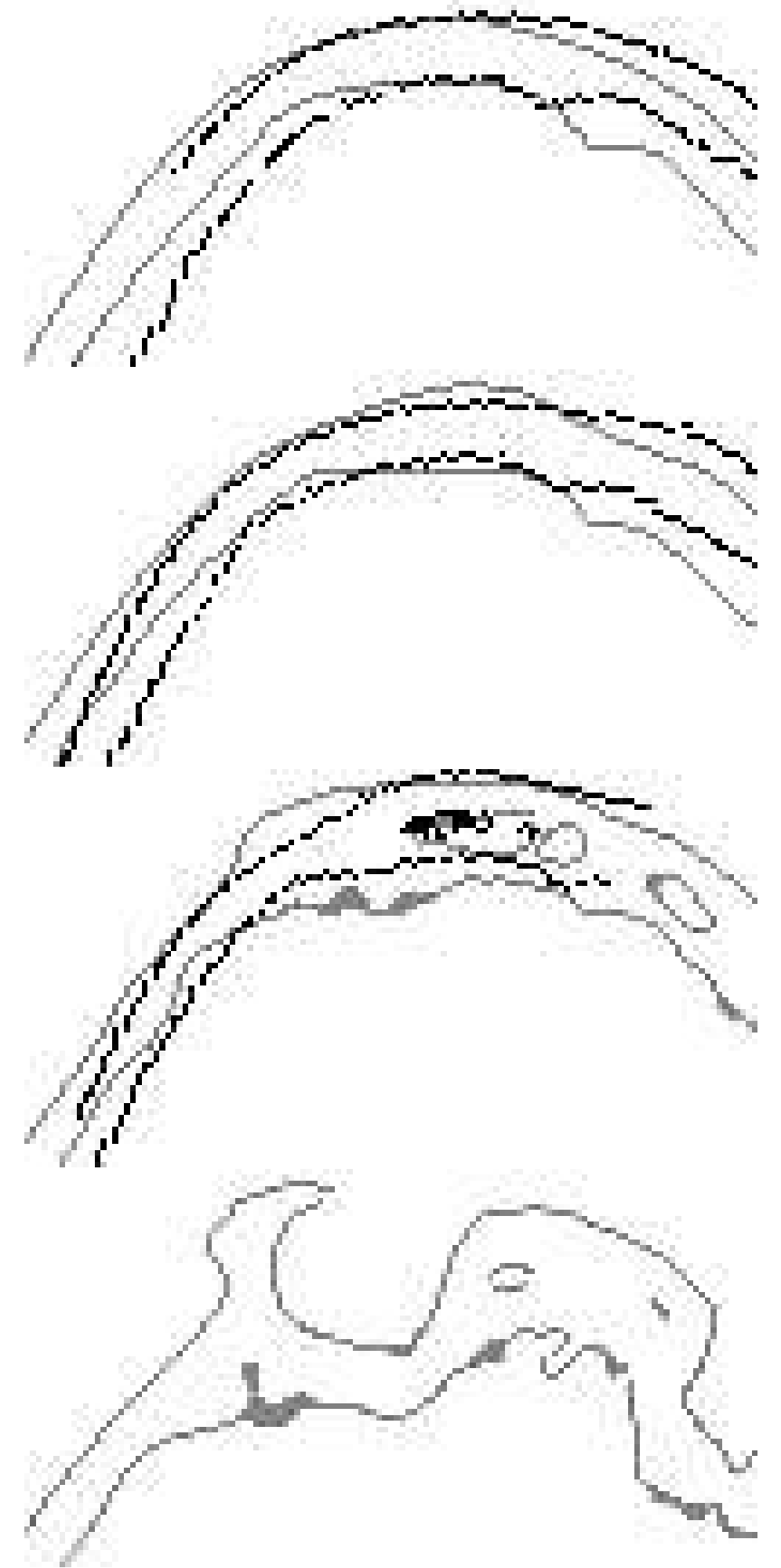

Figure 18: Grey points : sections from global structure - Black points : sections from target substructure (forehead) after recognition and locating. 
Our algorithm is then able to match each of the 5 patches in the substructure with the corresponding patch in the global structure; the 2 additional patches of the global structure are left apart. The locating result is shown in figure 18.

\subsubsection{Eye}
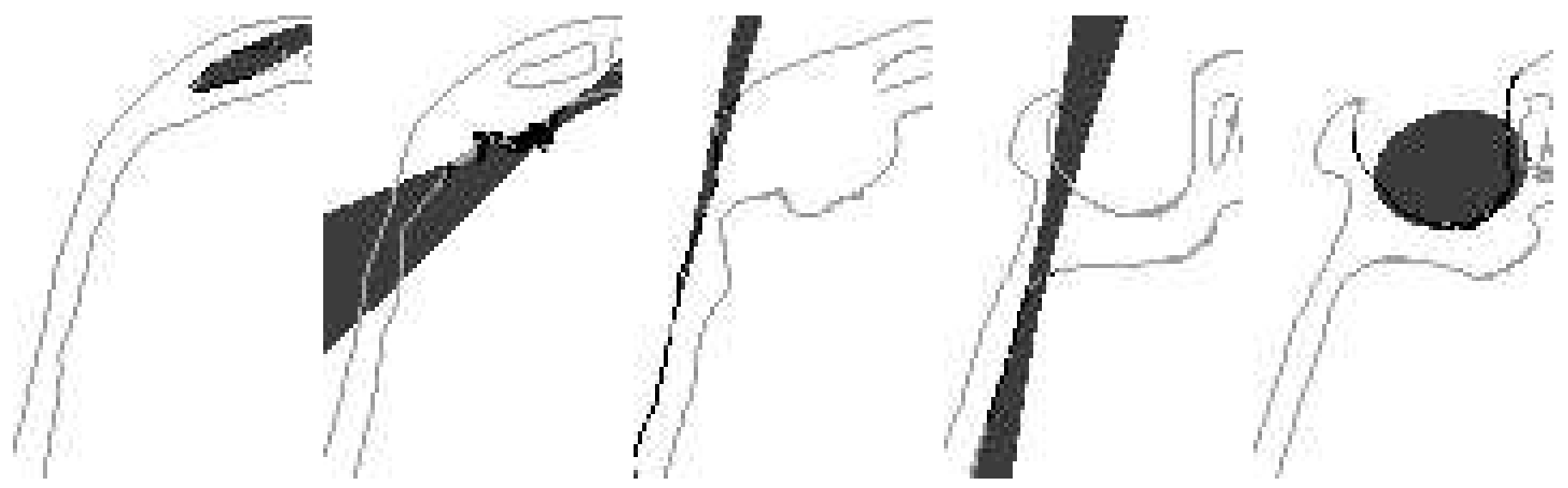

Figure 19: Eye -5 sections from the target substructure.

Here, a volumic scanner image including 28 successive sections defines the target substructure (the left eye). Five sections are reproduced figure 19. Using our quadratic patches extraction algorithm, we obtained 5 patches of important size. Each of these patches is represented in one section figure 19.

Our algorithm is then able to match each of the 5 patches in the substructure with the corresponding patch in the global structure. The locating result is shown in figure 20 .

\section{Comments}

From the quadratic segmentation results presented in figures 16,17 and 19 , we can point out the following facts :

- Edge images of the studied structures (forehead, skulll, eye) contain up to 13,000 points. Before the region-growing process, the initial segmentation contains up to 2,500 patches, whose size is very small.

RR $n^{\circ} 2380$ 

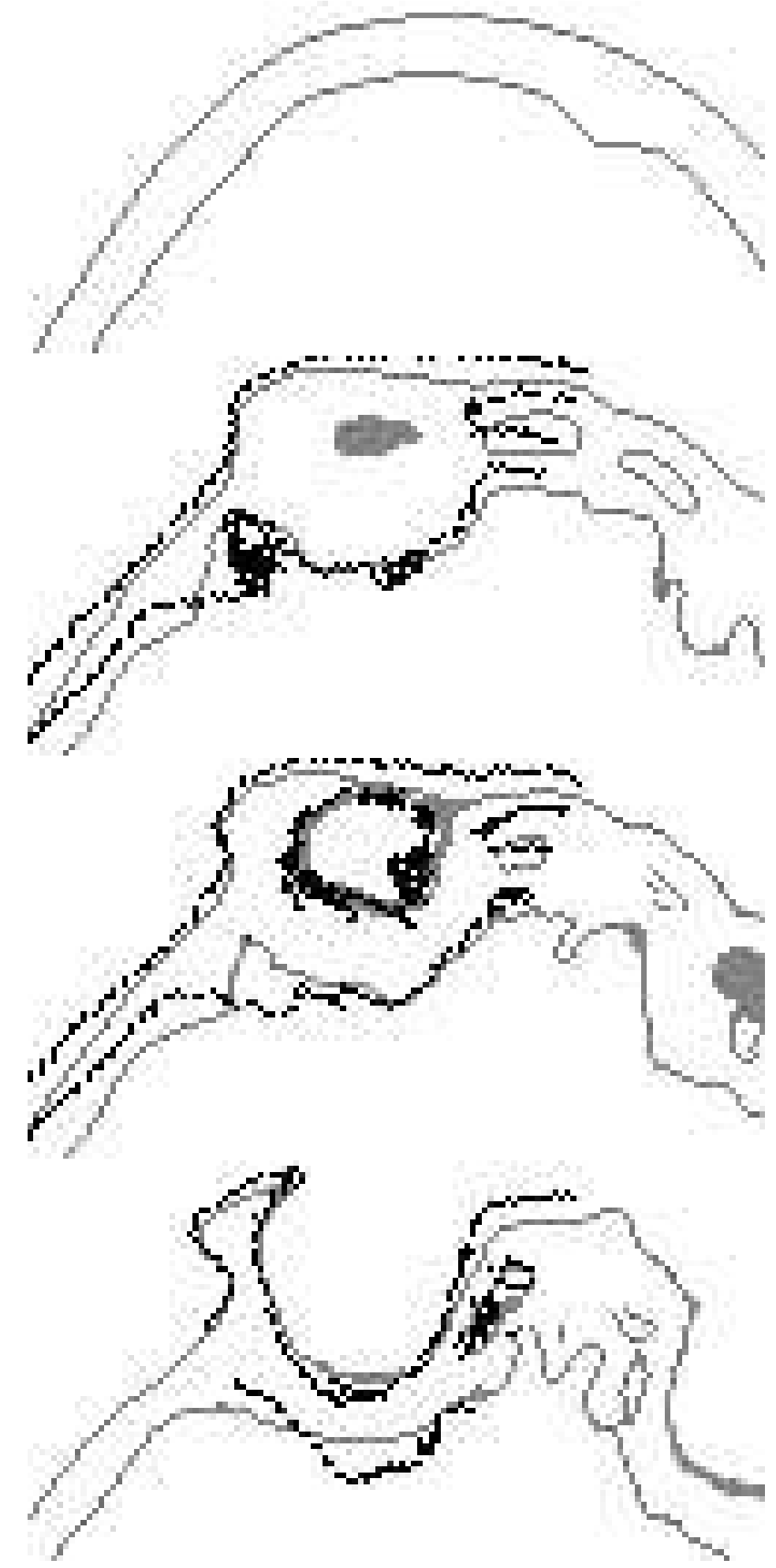

Figure 20: Grey points : sections from global structure - Black points : sections from target substructure (eye) after recognition and locating. 
- The segmentation of these 3 structures used the same threshold values.

- After the region-growing process, we automatically selected the few patches with more than 400 points. Despite hundreds of mergings necessary to obtain these patches, we can observe that they are similar among the various images.

- We can notice that the quadratic surfaces extracted are meaningful from an anatomic point of view. For instance, we can get informations about forehead shape or sinus size.

Besides, we have been able to perform rapidly the recognition and approximative locating of a substructure (a set of 8,000 edge points) on a global structure (a set of 13,000 edge points). This is possible with reduced computational time, because the quadratic segmentation has extracted and condensed a pertinent information about the studied objects.

\section{Conclusion}

This work is the natural continuation of the ones described in the references $[?, ?, ?, ?]$. The goal of these works is to define from original data (volume images) a hierarchical sequence of shape representations that may be used at each level to solve a given task such as registration, localization, recognition... In this paper we have adressed the problem set by the determination of intrinsic parametric surface models using the differential characteristics.

We used the key idea of reference [?] ie to approximate a surface using quadratic patches thanks to a graph based region growing strategy. Our contributions with respect to [?] are :

- We take into account the differential characteristics of the studied object (gaussian curvature and extremality), for the initial segmentation and during the merging.

- We implement, thanks to an adapted data structure, a fast optimal region growing algorithm.

- We introduce merging criteria that are specific (continuity, frontier points) and flexible (relative thresholds).

$\operatorname{RR} n^{\circ} 2380$ 
- The relevance and stability of the quadratic segmentation obtained is, as far as we know, without equivalent in previous works. The use of this quadratic information has allowed us to achieve efficient recognition and locating of real anatomic structures.

\section{References}




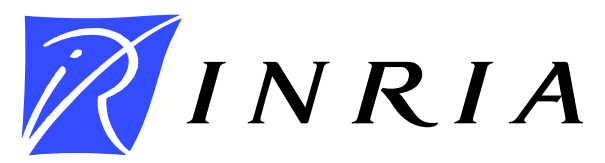

